\title{
SIMULATION ON VESSEL INTELLIGENT COLLISION AVOIDANCE BASED ON ARTIFICIAL FISH SWARM ALGORITHM
}

\author{
Weifeng LI \\ Wenyao MA \\ Navigation College, Dalian Maritime University, Dalian 116026, China
}

\begin{abstract}
TAs the rapid development of the ship equipments and navigation technology, vessel intelligent collision avoidance theory was researched world widely. Meantime, more and more ship intelligent collision avoidance products are put into use. It not only makes the ship much safer, but also lighten the officers work intensity and improve the ship's economy. The paper based on the International Regulation for Preventing Collision at sea and ship domain theories, with the ship proceeding distance when collision avoidance as the objective function, through the artificial fish swarm algorithm to optimize the collision avoidance path, and finally simulates overtaking situation, crossing situation and head-on situation three classic meeting situation of ships on the sea by VC++ computer language. Calculation and simulation results are basically consistent with the actual situation which certifies that its validity.
\end{abstract}

Keywords: simulation; collision avoidance; artificial fish swarm algorithm

\section{INTRODUCTION}

According to the survey, more than $80 \%$ of ship's collision accidents are related to human factors. There are two ways to solve the human factor. First, strengthen the skills of training and management abilities of ship's crew which will improve their quality. Second, implementing the automatic navigation and improving the automatic level of decision-making which can avoid errors caused by the judgment of human. As the development of technology, bridge resources provide more and more navigational information. If crews are not fully trained, a lot of navigational information may cause them to make incorrect judgments and decisions. And if crews make the wrong decision, it may lead to huge losses. Therefore, an effective method to reduce the human factor is to use high technology to improve the automatic level of navigation, which will reduce the crew's subjective judgment as well as the burden of the officer on the watch (OOW). Thus, the research on automatic collision avoidance decision system has practical significance for the safety of the ship.

Although automatic radar plotting aids (ARPA) can solve parts of problem about information processing of ship collision avoidance. It is not a fully automatic collision avoidance system. The OOW use it based on their subjective judgment of the experience and professional skills. Electronic Chart Display and Information System (ECDIS) can obtain navigational information and exchange the data and information by connecting other nautical navigation devices such as GPS (Global Position System), AIS (Automatic Identification 
System), radar, compass, log, VDR (Voyage Data Recorder), which is becoming a new type of ship navigation system and decision support system, namely ship navigation information system, and it gradually becomes the information core of the ship bridge. Ship collision avoidance strategy is a complex decision-making process, which includes sailing data collection, data preprocessing, division of vessel meeting situation, calculation of collision risk degree, selection method of collision avoidance, and the optimization of collision avoidance (Bai Yiming, 2012). Therefore, it is very difficult to describe ship collision avoidance with the precise mathematical model. Even with a very precise mathematical model, it is impossible to require real-time collision avoidance decision environment. Therefore, in recent years, scholars have begun to bring artificial intelligence methods such as artificial neural networks, fuzzy logic, genetic algorithms, ant colony algorithm in the field of ship collision avoidance (Lee S.M, 2004).

As the ship collision avoidance decision is a nonlinear problem with multiple indexes. It pursues not only the safety but also economic consumption. This study uses artificial fish swarm mixture optimization algorithm which different from the mathematical model. The algorithm imitates fish's forage, clusters rear-ends behavior and searches for the optimal solution. The algorithm can be used to solve highly complex engineering problems. Therefore it is suitable to ship collision avoidance route planning. Combining the international regulations for prevention collisions at sea (COLREGs 1972) and the safety domain of a ship, the algorithm can be used to get the most recommended ship collision avoidance path. This study combines ECDIS platform and forms the ship automatic collision avoidance decision support system which provides automatic route monitoring, collision warning and collision avoidance decision support prompts.

\section{SHIP DOMAIN AND MEETING SITUATION DIVISION}

Ship domain is an effective regional areas surrounding the ships which other ships and stationary targets should keep outside, and it is required waters to maintain safe navigation of any ship. Ship domain is an important concept of maritime traffic engineering, which widely used in ship collision avoidance and risk assessment. Fujii, Goodwin and Wu Zhaolin studies on the ship domain details(Fujii Y, 1971). In this paper, the collision avoidance decision supporting system requires that the target ship need to pass out of the ship domain.

According to the COLREGS, meeting situations can be divided into three types:

(i) Head-on situation: Target vessel approaches from Figure $1 \mathrm{~F}$ area. Own ship and target ship are meeting on reciprocal or nearly reciprocal courses so as to involve risk of collision and each shall alter her course to starboard so that each shall pass on the port side of the other.

(ii) Crossing situation: Target ship approaches from Figure 1 A, B, E area. Own ship and target ship are crossing so as to involve risk of collision, the vessel which has the other on her starboard side shall keep out of the way.

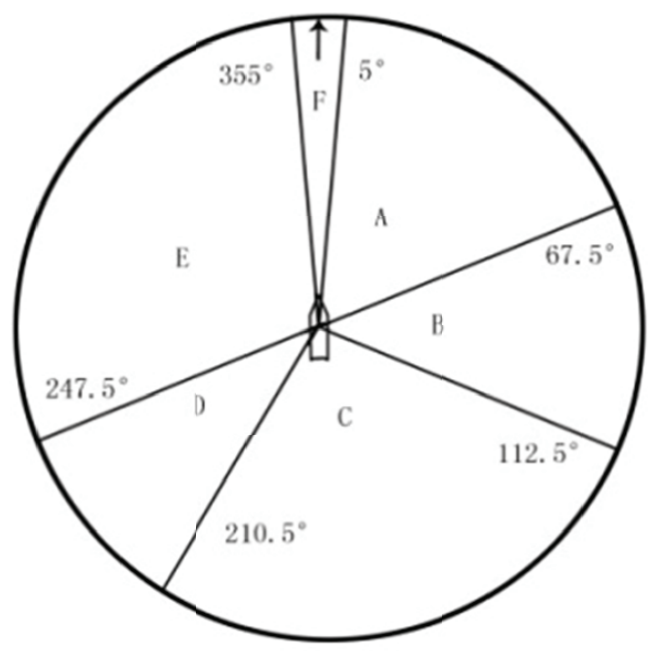

Fig 1. Division for ship meeting situation

(iii) Overtaking situation: Target ship approaches from Figure $1 \mathrm{C}, \mathrm{D}$ area. The ship shall be deemed to be overtaking when coming up with another ship from a direction more than 22.5 degrees abaft her beam. Overtaking vessel shall keep out of the way of the vessel being overtaken.

\section{MODELING OF COLLISION AVOIDANCE ROUTE PLANNING SYSTEM}

When two vessels are meeting, and the own vessel and the target vessel keep their original course and speed. When the target ship goes into the observation distance (observation information of the target vessel comes from own ship's AIS equipment), the direction of relative movement, distance to closet point of approach (DCPA) and time to closet point of approach (TCPA) will be got. The COLREGS established a knowledge guidance, which can be used to determine which meeting situation is forming between own ship and the target ship, and which ship is the give way vessel. If risk of collision exists and own ship is the give-way vessel, the study of decision supporting system will provide a collision avoidance route planning and recommend a safe and economical route. In fact, the route may not be the most feasible route, but the system theoretically can guarantee a safe and economical recommended route at least, which will contribute to early warning and decision supporting. Therefore, the OOW can use this route as a reference for the use of collision avoidance scheme.

According to the different mission phases, avoidance route is divided into three phases:

(i) Warning stage.

When the target ship goes into the observation and tracking phase, it needs to determine the meeting situation of the own ship and the target ship. It needs to determine whether risk of collision exists according to the target vessel's DCPA, if it 
less than the safety domain of own vessel, risk of collision exists. If the risk of collision exists and the own ship is giveway vessel, collision avoidance route planning algorithm will work. This stage, the algorithm will give the latest turning point of collision avoidance and maintain the original course and speed sailing sustainable time. The OOW can serve as a reference for the drawing up collision avoidance decisions.

(ii) Collision avoidance stage.

After steering for some time, the vessel sails into the stage of collision avoidance stage. The vessel altering course action should not be too small, otherwise, it's intention would be not easy to be found. If the altering course action is too large, it will result in the own vessel deviates too far from the original route. However, this angle must ensure that the target ship passes out of the security area of the own ship.

(iii) Course recovery stage.

The time to recovery her original course and the altering course angle should guarantee that two vessels will not form a new hazardous situation in the course recovery stage.

\section{COLLISION AVOIDANCE ROUTE PLANNING BASED ON ARTIFICIAL FISH SWARM ALGORITHM}

\section{THE PRINCIPLE OF ARTIFICIAL FISH SWARM ALGORITHM}

The principle of artificial fish swarm algorithm is to simulate natural fish's forage, clusters, rear-ends behavior and mutual assistance between fish to achieve global optimization (MingCheng Tsou, 2010). Artificial fish foraging behavior is the random walk based on the current value of their adaptation, it is an individual extreme optimization process and selflearning process. The fish's clusters and rear-end behavior is the interaction with the surrounding environment. The conduct of algorithm is self-adaption process for artificial fish, the process includes fish's forage, clusters and rear-ends behavior, and the optimal projection emerges in the process. Thus, artificial fish swarm algorithm is a kind of optimization method based on swarm intelligence. The optimization process of artificial fish makes full use of their information and environmental information to adjust its search direction, and ultimately searches to the highest places of food concentration, namely the global extreme value.

Therefore, artificial fish swarm algorithm is an effective global optimization intelligent algorithm, which has a unique and superior performance compared with other traditional optimization methods for some complex optimization problems.

\section{ARTIFICIAL FISH INDIVIDUAL CODING SCHEME}

There are four parameters of collision avoidance system should be encoded.

(i) The time from current position to the position of turning point to avoid collision, $T_{S}$ : altering course must be carried out within the $T_{S}$. The altering course action must be executed at least when the ship is at turning poin, otherwise the risk of collision exists.

(ii) The minimum altering course angle $\Delta C_{O}$, which indicates that the ship can pass with a safe distance with the target ship when altering this angle. The actual altering angle should be not less than $\Delta C_{O}$; otherwise the risk of collision exists.

(iii) The time from taking altering course action ther original course recovered, $T_{a}$. This parameter indicates that the ship must navigate with $T_{a}$. minutes before her original course recovered.

(iv) Course changing amount when the ship taking action to her original course, $\Delta C_{b}$. It is the maximum angle the ship needs to change when taking action to come back to her original course.

Four variables of collision avoidance route optimization decision corresponds to the artificial fish individual $\theta_{i}=\left[T_{s}, \Delta C_{O}, T_{a}, \Delta C_{b}\right]$

\section{THE OBJECTIVE FUNCTIONS}

The distance from the point the ship is taking action to avoid collision to the point the ship's course recovered, which can be used to assess the route planning. Therefore, it can be used as the objective function. The paper tries to get the shortest path to avoid collision with artificial fish optimization algorithm and makes the following constrains.

(i) Collision avoidance distance should be minimal;

(ii) Risk of collision should be minimal and, own ship and target ship should pass at a safe distance;

(iii) The altering course angle should be minimal;

(iv) The track should be minimal during the circuitous voyage when the ship is taking collision avoidance action;

(v) The course changing amount should be minimal if there is no other new meeting situation or urgent situation.

Assuming that the target vessel course $C_{T}$, speed $V_{T}$, relative to the own vessel's position $\mathrm{Q}$, distance $d$, the own vessel course $C_{O}$, speed $V_{O}$, and after avoiding collision action the new course $C_{O}^{\prime}$, then the objective function is:

$$
J=\min \left\{d_{s}+d_{r}\right\}
$$

$d_{s}$ is the own vessel steering distance during the collision avoidance, $d_{r}$ is the own vessel steering distance during the the process of ship's course recovered.

$$
\begin{gathered}
d_{s}=T_{a} \cdot V_{O} \\
d_{r}=T_{a} \cdot V_{o} \cdot \sin C_{o}^{\prime} / \sin C_{b}^{\prime} \mid
\end{gathered}
$$

$C_{O}^{\prime}$ is course changing amount when collision avoidance. $C_{b}$ is course changing amount when the ship is coming back to her original course. $T_{\mathrm{a}}$ is the time from taking altering course action t0 her original course recovered. The constraint condition is shown as function(4). 


$$
\left\{\begin{array}{l}
30 \leq C_{O}^{\prime} \leq 90 \\
-60 \leq C_{b} \leq-30 \\
T_{C P A 1} \leq T_{a} \leq 60 \\
\left(d_{C P A 1} \leq d_{G}\right) \text { and }\left(d_{C P A 2} \geq d_{G}\right)
\end{array}\right.
$$

$C_{O}^{\prime}$ is course changing amount when collision avoidance, positive values indicate right turn; $C_{b}$ is course changing amount when the ship is coming back to her original course, negative values indicate the left turn; $T_{\mathrm{a}}$ should not exceed 60 minutes and must be at least more than the time of encountering $T_{C P A 1}$ (the new recent time after collision avoidance action); $d_{C P A 1}$ and $d_{C P A 2}$ are distance to closet point of approach after collision avoidance and recovery of her original course.

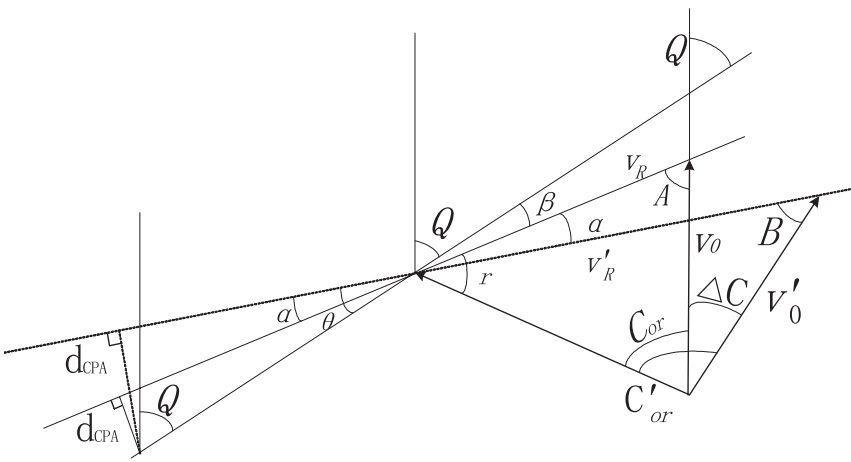

Fig.3 Collision avoidance decision modal after the home ship is turned

$d_{C P A}$ is distance to the closest point of approach and $T_{C P A}$ is time to closest point of approach are key factors meeting the objective function limiting conditions, which can be calculated according to the literature mentioned methods. Figure 3, newd $d_{C P A}$ and $T_{C P A 1}$ can be calculated in the following method after the preventing collisionand steering:

$$
\left\{\begin{array}{l}
d_{C P A 1}=d * \sin \theta \\
T_{C P A 1}=d * \cos \theta / V_{R}^{\prime}
\end{array}\right.
$$

$\theta$ is the angle between the relative motion line of the own vessel and the bearing line of the target vessel; $d$ is the distance between the own vessel and the target vessel; $B$ is the angle between the relative motion line of own vessel and the route of the own vessel after taking collision avoidance action; $C^{\prime}{ }_{O T}$ is the angle between the own vessel and the target vessel after steering; $V^{\prime}{ }_{R}$ is the relative velocity of the own vessel after steering.

$$
C_{O T}^{\prime}=C_{0}^{\prime}-C_{T}
$$

$$
V_{R}^{\prime}=\sqrt{V_{T}^{2}+V_{0}^{2}-2 * V_{T} V_{o} \cos C_{O T}^{\prime}}
$$

if $C_{O T}^{\prime} \geqslant 0^{\circ}, \theta=B+C_{O}^{\prime}-Q$;

$$
B=\cos \left(\left(V_{0}^{2}+V_{R}^{2}-V_{T}^{2}\right)^{2} / 2 V_{0} V_{R}^{\prime}\right)
$$

$$
B=-\cos \left(\left(V_{0}^{2}+V_{R}^{2}-V_{T}^{2}\right)^{2} / 2 V_{0} V_{R}^{\prime}\right)
$$

$d_{G}$ is radius of safe passing circle; and the new $d_{C P A}$ should be more than the radius at least. The value depends on the maritime traffic environment and ship type.

\section{SIMULATION STEPS}

Step 1. Initialization of groups. Randomly generated $N$ artificial fish individuals in the variable feasible region, and got the initial fishes. Setting artificial fish visual field Visual, the maximum moving step length step, crowding factor $\delta$, the biggest temptation for each mobile number trynumber. Setting the iteration number of initial bulletin board optimal state of not changing or little changing of optimal artificial fish state Beststep $=0$, the initial iteration $\mathrm{Num}=0$.

Step 2. Initialization of bulletin board. Calculate the initial fish each artificial fish the objective function value and compare the size of it, whichever is the optimal state and its value is assigned to the artificial fish bulletin board.

Step 3. Behavior selection. Simulate rear-end behavior and swarming behavior for each artificial fish, choose the best behavior and perform by comparing the value of the objective function, the default behavior is foraging behavior.

Step 4. Update Bulletin Board. Each artificial fish compares their own function value and bulletin board value, if their own function value is better bulletin board value, bulletin board value is replaced, otherwise the value of the bulletin board is unchanged.

Step 5. Introduce genetic algorithm to conditional judgement. If Beststep has reached the preset consecutive not changing the maximum threshold Maxbest, genetic algorithm crossover and mutation algorithm of Step 6 is executed, otherwise, go to Step 7.

Step 6. The operation of genetic algorithm crossover and mutation. all other artificial fish do these operation except the best individual bulletin board: (1) crossover: Randomly select a number of individual fish from artificial fish based crossover probability $\mathrm{P}_{c}$, divide into group and execute the operation of crossover for two individual fish. Compare function calculated of new individual fish formed to the optimal value of the bulletin board, if it is better than the bulletin board's value, the bulletin board's value is replaced, and the new instance replace the old individuals. (2) mutation: Randomly select a number of individual fish based on the mutation probability $\mathrm{P}_{\mathrm{m}}$, execute the operation of the mutation of these individuals. Calculate function value of newly formed artificial fish, and in comparison with the optimal value of bulletin board, if it is better than the value of the bulletin board, then it replaces the value of the bulletin board. (3) set Beststep $=0$.

Step 7. Terminate conditional judgment. Repeat steps Step 3 6 until the optimal solution to achieve the bulletin board's satisfactory error bounds.

Step 8. Terminate the algorithm. Output the optimal solution (artificial fish bulletin board's status and its function values).

if $C_{O T}^{\prime} \leqslant 0^{\circ} \quad \theta=-\left(B+C_{O}^{\prime}-Q\right)$ 


\section{SIMULATION RESULT}

The algorithm decision supporting system settings and results are shown in Figure 4. Ship's dynamic information is from the AIS, and the vessel speed is $14 \mathrm{kn}$, initial course is $000^{\circ}$, and course changing can be performed only when to avoid collision. Target vessel speed is $15 \mathrm{kn}$. Vessel's collision avoidance can be divided into head-on situation, overtaking situation and crossing situation according to the COLREGs. Simulating the algorithm by following three typical cases:

(I) The own vessel is crossing encountering with right frontage of the target vessel.

(II) The own vessel is crossing encountering with right rear of the target vessel.

(III) Head-on situation.

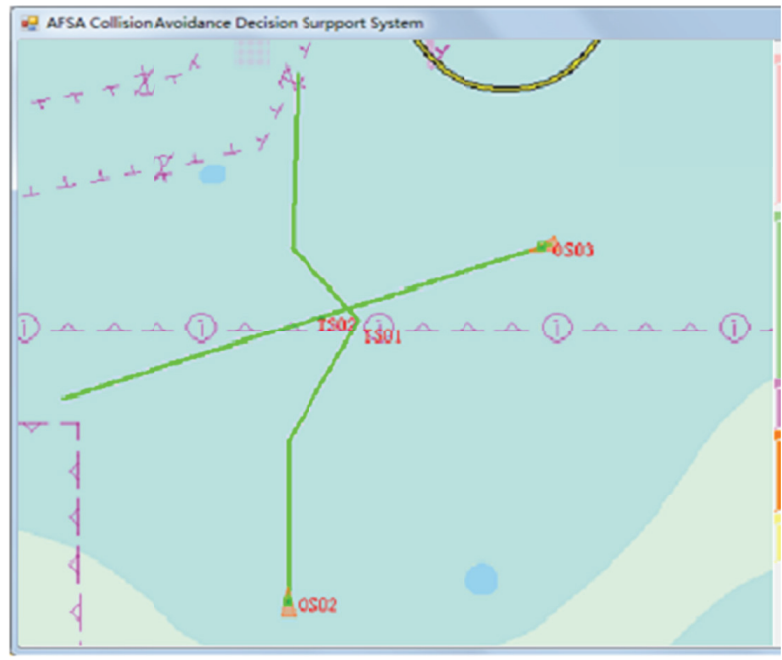

Fig 4.Decision supporting system for collision avoidance route planning

Table 1 shows the simulation results and route planning data above three cases by artificial fish swarm algorithm. $d_{C P A}$ is the distance to closet point of approach and $t_{C P A}$ is time to closet point of approach in initial state. In this table, $d_{C P A}>0$, the target vessel passes by the bow of the own vessel; $d_{C P A}<0$, the target vessel passes by the stern of the own vessel. $T_{1}$ is time to turning point, which means that the moment of reaching $T_{1}$ begins to steer from the observed moment calculated, $C_{1}$ is steering angle to the right avoiding collision, it is limited $[30,60]$ within the range, if the angle is less than $C_{1}$, then the risk of collision exists. $T_{2}$ represents that the vessel should sail $T_{2}$ minutes with new course after avoiding collision and steering. In order to ensure the safe passage of the target vessel; $\mathrm{C}_{2}$ represents the altering course angle to recovery her original course, if the altering course angle is too large, it may cause a new risk of danger with the other vessels in the vicinity.

The simulation suggests that the improved artificial fish swarm algorithm can give the optimal collision avoidance route, which is both safe and economy. When connecting with ECDIS, collision avoidance parameters can be dynamically displayed in the ECDIS platform, which can provide decision supporting to avoid collision and can effectively reduce the burden of the OOW and improve the safety of traffic on the sea.

\section{CONCLUSION}

The study combines safety domain of ship and the COLREGs, adopts artificial fish optimization model to optimize the key parameters of collision avoidance decision model and forms a collision avoidance decision supporting system, which can quickly provide the OOW a safe and economical collision avoidance route. Although the study uses the instance of the single target vessel avoidance as a demonstration, the decision optimization algorithm is suitable for multi-target vessel avoidance situations. Once the system connects with the bridge navigational equipment and ECDIS, it will provide some security and support for collision avoidance at sea and VTS monitoring waters.

\section{ACKNOWLEDGEMENTS}

This work was financially supported by the Fundamental Research Funds for the Central Universities with number of 3132015009.

\section{REFERENCE}

1. Lee, S.M., Kwon, K. Y. and Joh, J. A fuzzy logic for autonomous navigation of marine vehicles satisfying COLREG guidelines[J]. International Journal of Control Automation And Systems, (2004).2, 171-181.

2. Egil Pedersen and Kinzo Inoue. Simulator Studies on a Collision Avoidance Display that Facilitates Efficient and Precise Assessment of Evasive Manoeuvres in Congested Waterways[J]. Journal of Navigation.2003(56):411-427.

3. Ming-Cheng Tsou, Sheng-Long Kao, Chien-Min Su. Decision Support from Genetic Algorithms for Ship Collision Avoidance Route Planning and Alerts[J]. Journal of Navigation, 2010, 63(1), 1-16.

4. Ming-Cheng Tsou, Chao-Kuang Hsueh. The Study of Ship Collision Avoidance Route Planning by Ant Colony Algorithm. Journal of Marine Science and Technology,

Tab.1.Parameters of collision avoidance of AFSA

\begin{tabular}{|cccccccccc|}
\hline $\left.\mathrm{C}_{\mathrm{T}}{ }^{\circ}\right)$ & $d_{\text {OPA }}(\mathrm{nm})$ & $t_{\text {OPA }}(\min )$ & $\mathrm{T} 1$ & $\mathrm{C} 1$ & $\mathrm{~T} 2$ & $\mathrm{C} 2$ & $\mathrm{Q}\left({ }^{\circ}\right)$ & $d(\mathrm{~nm})$ & $d_{s}(\mathrm{~nm})$ \\
\hline 246 & 0.74 & 73 & 67 & 47 & 13 & -46 & 41 & 28 & 5.85 \\
286 & -0.22 & 80 & 61 & 53 & 15 & -55 & 80 & 23 & 6.78 \\
190 & 0.42 & 66 & 52 & 45 & 13 & -37 & 2 & 33 & 6.32 \\
\hline
\end{tabular}


5. Rafal Szlapczynski. A Unified Measure Of Collision Risk Derived From The Concept Of A Ship Domain[J]. Journal of Navigation.2006(59):477-490.

6. Jang San, Je Yefei, Dasgupta S. Artificial Fish Swarm Algorithm for Solving Road Network Equilibrium Traffic Assignment Problem [J]. Journal of computer emulation,2011,28(6): 326-329.

7. Pedersen.E, Inoue .K, Masanori. T. Simulator Studies on a Collision Avoidance Display that Facilitates Efficient and Precise Assessment of Evasive Manoeuvres in Congested Waterways [J]. The Royal Institute of Navigation (2003). 411-427.

8. Bi Xiuying, Jia Chuanying, Wu ZhaoLin. Opportunity and Actions Taken of Ship's changing speed collision avoidance. Journal of Dalian Maritime University, 2003, 29(1):9-12.

9. Liu Dexin, Wu ZhaoLin and Jia ChuanYing. Decision Making Model of DCPA, TCPA and Object's Movement Parameter . Journal of Dalian Maritime University, 2004, 30(1), 22-25.

10. Zhao Yuelin. Ships collision avoidance and watch keeping[M].Dalian Maritime University,2012.

11. Gemeinder M, Gerke M. GA-Based Path Planning for Mobile Robot System Employing an Active Search Algorithm [J]. Applied Soft Computing, 2003,(3):149-158.
Weifeng LI

sddmlwf@163.com

Navigation College

Dalian Maritime University

Dalian 116026

CHINA 